\section{MEDICAL ASPECTS OF CHRONIC PEPTIC ULCER}

By R. Sleigh Johnson, M.D., M.R.C.P. (Physician, London Chest Hospital, Southend General Hospital, and Royal Waterloo Hospital)

CHAPTER I

General Considerations

Pathology and Pathogenesis

Symptoms and Physical Signs

Diagnosis and Special Investigations

CHAPTER II

Treatment

\section{Chapter I \\ Chronic Peptic Ulcer}

\section{General Considerations}

Few diseases, whether from their commonness or their difficulties in treatment, can claim a more constant place in medical attention than peptic ulcer, a comment applying with equal force to physician and to surgeon, to hospital and to general practice. Figures of emergency admissions show few conditions higher on the medical side than acute gastric haemorrhage, while surgical complications demand a like proportion. In such an ever-present disease, it is a disturbing thought that despite a welter of clinical study and experimental work, the aetiology should defeat exact investigation and remain largely obscure, and perhaps no less disturbing that so little uniformity of agreement should have been reached on policies of treatment, particularly in stages where medical and surgical aspects overlap. In consequence the results of treatment of peptic ulcer cannot yet be claimed to be satisfactory, whether ensuing either from medical zeal or from surgical enthusiasm, and an attempt to review and co-ordinate current opinion may not therefore be out of place.

\section{Aetiology}

In distribution peptic ulcer is world-wide without respect of race, occupation, or class, and occurs within a wide range of ages. From autopsy findings it is estimated that at some period in his life one person in ten is so affected.

The age group most commonly involved lies between 20 and 40 years, but acute ulcers revealed by haemorrhage are met with in infancy or early childhood.

A familial factor is often easily traced.

It is probable that many more gastric ulcers than duodenal are relatively symptomless, and autopsy findings show a higher incidence of healed ulcers in the stomach, but from the clinical stand- 2 point duodenal ulcer is much the commoner $\frac{3}{\infty}$ disease, in proportions of at least three to one, $\stackrel{\varrho}{c}$ and correspondingly more resistant to treatment.

Regarding the sex incidence, duodenal ulcer $\overrightarrow{\bar{F}}$ is between three and four times commoner in $\frac{7}{0}$ males, whereas gastric ulcer is relatively more $\frac{0}{\sigma}$ frequent in females. From two aspects, physique $\frac{\overline{\bar{D}}}{\bar{D}}$ and mental make-up, an "ulcer type" may be $\vec{\nabla}$ readily recognised in the spare lean build and anxious over-active manner, the more so when $s$ the site is duodenal. It occasions no surprise that $\overrightarrow{0}$ the incidence of peptic ulcer appears to have $\overrightarrow{ }$ risen considerably during the past thirty years, in $\vec{\sigma}$ response to strain and stress of living and the increased pace of life; part of this apparent rise $\frac{O}{3}$ is no doubt the result of improved facilities for diagnosis, but after allowance for this there is $\overrightarrow{-}$ certainly a real increase, particularly in the frequency of duodenal ulcer. The greater incidence of this lesion in men is reflected anatomically in $\ddot{8}$ the hypertonic type of stomach known to pre-o dominate in the male, with its hypersecretion and rapid rate of emptying, whereas simple gastric $\vec{c}$ ulcer is less constantly linked with hyperacidity and excess of tone, and is therefore by no means ${ }^{D}$ rare in the asthenic anaemic type of woman witb $\overrightarrow{0}$ low-lying or hypotonic stomach. This distinction of of type is further emphasised in the differem psychological and emotional background of the two groups.

Occupational factors in aetiology are inconstant. Whereas a type of employment demanding physi- $\frac{\circ}{\square}$ cal rush and mental strain, with hasty and irregular meals, no doubt contributes to break-down in those predisposed, peptic ulcer is common enough among all classes of occupation and society. Although ulcer symptoms, including perforation, not infrequently follow nervous stress, anxiety or emotional upset, these are probably contributory rather than causative factors. Onset is 3 . often insidious and difficult to date, and accentuation by such factors may readily be mistaken $\frac{0}{3}$ for start of the disease.

The relation of tobacco and alcohol to peptic $\stackrel{\text { ? }}{?}$ ulcer is similar; while there is no proof that smoking, $\frac{D}{0}$ even in excess, is a direct causative factor, it is generally agreed that the swallowing of nicotine $N$ excites free secretion of gastric juice, which can only be undesirable and harmful to an empty stomach.

Teeth which are carious or infected, or are deficient in number, impair the preparation ofo food for gastric digestion and may through sepsis set up a contributory gastritis.

Acute infections of any kind may act in a similar $\frac{0}{0}$ way to nervous worry, fatigue, or rushed or un- $\frac{\vec{D}}{\mathbb{D}}$ 
suitable meals, in precipitating the acute exacerbations so characteristic of the disease.

\section{Pathology and Pathogenesis}

Peptic ulcer is usually a single lesion but separate ulcers are not uncommon in stomach and duodenum, or more than one ulcer may be present in the stomach. Similarly active and healed lesions may co-exist, or a single ulcer may show extension and healing in different directions at the same time, although one of these processes is usually predominant. The region liable to peptic ulceration corresponds accurately with the extent of exposure to the acid gastric secretion. The great majority of ulcers are included within the lesser curvature of the stomach, particularly its central third, or within the first two inches of the duodenum, the duodenal cap or bulb; the pylorus is frequently involved and its function affected by a pre- or post-pyloric ulcer. For the same reason a characteristic lesion is common in the area of jejunum directly opposed to a patent gastro-enterostomy, while rarely a typical peptic ulcer may develop in ectopic gastric mucosa at the lower end of the oesophagus or in a Meckel's diverticulum. The greater curvature of the stomach is a rare site for benign ulceration, and this situation alone should arouse suspicion of malignancy.

Opportunities for direct study of peptic ulceration in its varying stages were formerly confined to operative or post-mortem inspection. Within the last few years, however, our knowledge of its life history and pathology has been greatly extended and clarified by the direct inspection in the living subject afforded by gastroscopy.

An acute stage necessarily precedes the development of every chronic peptic ulcer, and is revealed as a sharply circumscribed loss of tissue beginning in the mucosa. This forms a shallow erosion, often small in size, with clean cut punched-out edges and smooth floor, sometimes covered by white or yellowish slough. Signs of inflammation of the surrounding mucosa may or may not be present, but at this stage there is no protective thickening of the peritoneal surface. Such an ulcer, if destruction is progressive, may rapidly penetrate all layers of the gut, unresisted by any barrier of fibrosis and terminate in perforation.

Healing, on the contrary, frequently takes place at this early stage by growth of a thin layer of mucosal cells across the defect, leaving either a normal looking surface or little more than a stellate puckering of the mucosa. Should the healing process fail, the ulcer extends both in surface area and by a progressive penetration into the deeper submucous and muscular layers of the viscus or beyond.
The chronic ulcer commonly becomes thickened and indurated by inflammatory connective tissue formation, with deep and terraced margins, in some cases undermined. The floor of such an ulcer may be clean in appearance and covered with granulation tissue, or concealed beneath a fibrinous exudate or slough, in which thrombosed vessels of considerable size may be situated. Adhesions to adjacent organs are of frequent occurrence in an ulcer of long duration, so that the liver or pancreas may come to form its base. Scarring from ulcers of marked chronicity may also lead to gross deformity and obstruction within the organ, such as pyloric stenosis or hour-glass stomach, while spasm and oedema may closely mimic organic occlusion.

The fluctuating life-cycle of a chronic peptic ulcer, reflected clinically in periodic remissions and exacerbations of symptoms, is found pathologically to correspond with phases of fresh ulceration and partial healing. Periods of quiescence or complete absence of symptoms tempt both patient and clinician to assume that the ulcer has healed, and pathological and X-ray tests may lend support to this erroneous conclusion, but direct inspection will frequently show that the lesion is merely inactive and dormant. What is often thought to be a fresh ulceration is but reactivity or breakdown of an old lesion. For this reasono also, a sudden unexpected haemorrhage or perforation may be the first indication that all is not as well as was thought.

Malignant transformation of benign gastric ulcer is a controversial and unsettled subject as to its frequency and even occurrence, opinion of its incidence varying from nil to ro per cent. of chronic simple ulcers. The writer's personal view is that it is a rarity, though its possibility cannot be denied. The common site of carcinoma in the stomach differs radically from that of simple ulcer; carcinoma practically never occurs in the first part of the duodenum, where as a sequel to long-standing irritation of chronic ulcer it would be frequently anticipated. Similarly unknown is malignant disease at a gastro-jejunal stoma. In the great majority of cases of carcinoma of the stomach, moreover, the age incidence affects an older period, and the history of digestive disorder is relatively short, the disease appearing clinically to start de novo rather than as a sequel to longstanding dyspepsia. It is recognised that owing to digestive changes the histological features of benign and malignant ulcer may be closely similar.

\section{Theories of Causation of Peptic Ulcer}

Many theories of causation of peptic ulcer have been advanced, and attempts made to repro- 
duce their effects experimentally. It is clear that they are unrelated to any specific bacteriology; the occasional association of acute ulcer with severe generalised infection, revealed for the most part post mortem, shows no common identity of organisms and, as in its rare sequel to burns, the ulcer here is probably a reaction to the absorption of tissue-breakdown or histamine products. Ulcers produced experimentally in animals by injection of organisms into the gastric mucosa show rapid healing and no tendency to progress to a chronic stage; traumatic ulcers of the stomach, both in the animal and the human subject, caused by the swallowing of a foreign body show a similar prompt resolution.

The frequent demonstration of thrombosed vessels in the base of a chronic ulcer has suggested that a local vascular spasm of the mucosal arterioles may be the primary factor, bringing about a loss of blood-supply to the area with necrosis of mucous membrane by infarction. Such a theory is at once disproved by the free anastomosis of vessels found in the stomach, wide ligature of which fails experimentally to produce ulceration; the thrombosis so often seen is the result and not the cause of the lesion. Similarly an attempt to explain the production of peptic ulcer by a neurogenic disturbance carries little weight; the sole neurological association which can be demonstrated in ulcer subjects is a vagal overaction, a reasonable explanation of the hypersecretion and hypermobility of the stomach which is present.

While the responsible factors for initiation of tissue loss remain obscure, the failure of healing of the breach of surface, when once begun, is less difficult to understand. There is present in these subjects with considerable constancy an inability of the mucous membrane to resist the digestive action of the acid gastric juice, a defect roughly proportional to the degree of hyperacidity present. Whether the ultimate cause is the excess of hydrochloric acid itself or some other abnormal condition of the gastric secretion is a moot point, for acidity by no means represents the sum-total of peptic activity. Again, whether the weak spot in defence is a lack of cellular resistance to digestion or a lack of cellular protection by mucus-secreting cells (a secretion lessened in ulcer subjects) is equally unknown. The important. point is that the presence of hydrochloric acid in excess is the main known factor in maintaining the activity of peptic ulcer.

Time-factors are of no less significance. Where, as in ulcer-subjects, the duration of exposure of the mucosa is prolonged, for example during the night or for long fasting hours between badly spaced meals, times during which the normal stomach would produce little or no acid, this abnormal digestive process is intensified.
This relation of hyperchlorhydria to peptic $\frac{6}{7}$ ulcer is confirmed in a number of ways. The site $\varrho$ of ulceration is confined strictly to those portions $\frac{\vec{D}}{\bar{\alpha}}$ of the digestive tract coming into direct contact $\varrho$ with the acid gastric secretion. Simple ulcer, $\stackrel{\complement}{.}$ on the contrary, is never found among the ro per $\overrightarrow{\bar{\omega}}$ cent or so of the population with a congenital $\underset{0}{\sigma}$ histamine-achlorhydria. Gastric ulcers produced $\frac{\sigma}{\sigma}$ artificially in animals fail to heal if the normal $\frac{\sigma}{\omega}$. neutralisation of acidity by duodenal contents is $\vec{\Phi}$ prevented, or if an abnormally high level of acidity is maintained in the stomach, either by direct $\omega$ administration of acid or by maintaining a hyper- $\overrightarrow{0}$ secretion by histamine injections.

Despite this evidence the question of acidity is $\vec{\omega}$ clearly not the whole story. Although a great $\frac{\sigma}{0}$ hypersecretion of acid (up to 0.4 per cent $\mathrm{HCl}$ ) $\frac{0}{3}$ is the common finding in peptic ulcer, especially of the duodenum, other patients will show normal $\vec{\sim}$ levels of acidity, and it has to be admitted therefore that the problem is still incompletely understood.

Of scarcely less importance in the aetiology of $\overrightarrow{0}$ peptic ulcer is the question of muscle tone and contractility. Duodenal ulcer subjects in particular commonly have a hypertonic stomach, $\vec{c}$ undergoing violent peristalsis with rapid emptying of acid secretions into the duodenum. In other $\mathbb{D}$ cases, pylorospasm will be the cause of a persistently $\vec{\varphi}$ high acidity of gastric contents from delay in the stomach emptying. Much stress has been laif. by gastroscopists upon the even closer anatomica relationship of ulcer incidence to hyper-rugosity of the stomach mucosa.

\section{Symptoms of Peptic Ulcer}

The outstanding symptom of peptic ulcer is pain related to the taking of food. This may be so characteristic in its features as to be practically diagnostic; two significant aspects are nearly always present, the long duration of the pain over months or years, and the periods of freedom from symptoms for a few days or more often weeks or months, during which the ulcer is quiescent but usually not healed. With progress of time the tendency is for the spells of pain to become more frequent and prolonged and often of $\rightarrow$ greater severity, with shorter intervals of freedom. 을 In character the pain is usually described as $N$ aching or gnawing, sometimes burning or colicky, varying from a mild discomfort to severe distress, and felt over a range of situations in the upper abdomen. It is felt most commonly in the central epigastrium, being often localised to a small area, but may be referred alternatively to one or other hypochondrium or to a combination of these sites. Diagnosis of the probable seat of ulceration from the point of reference of pain is of little or 
no value, although duodenal pain is more commonly felt to the right of the abdomen. Radiation of the pain to the back or around the right costal arch, especially when it is severe and unrelieved by the usual measures, often indicates a deep, penetrating ulcer adherent to the posterior abdominal wall or pancreas. Pain from a jejunal ulcer after gastroenterostomy is apt to be experienced in the left mid-abdomen or below.

The daily rhythm of the pain, as distinct from its longer periodicity, is shown in its clockwork regularity after the taking of food, at an interval varying from a few minutes to three hours or more. The heavier the meal the more severe the pain, which may sometimes only follow the main meal of the day. As with site, the time-relation of the pain is no certain guide to the situation of the ulcer. In general, however, pain from duodenal ulcer occurs at a long interval after food, which it often appears to precede rather than to follow. It is an especial feature in the small hours of the morning, waking the patient from sleep. Relief in uncomplicated ulcer is afforded with considerable constancy by alkalis or a further meal, or by vomiting, while partial relief is obtained by rest. The almost immediate alleviation by radical dieting is so marked in many cases as to confirm the diagnosis. The association of the pain with craving for food and the relief so given leads to its apt designation as "hunger-pain". In mechanism of production, pylorospasm and irregular muscular contractions are partly responsible; there are also the factors of increased sensitivity of the mucosa to mechanical or chemical stimuli, e.g. a raised hydrogen ion concentration or inflammatory changes in or around the ulcer. The normal gastric or duodenal lining is insensitive and hyperacidity alone does not produce pain; acidity in a given case shows little variation in degree whether the ulcer is active or healing, but a lowered threshhold of sensation causes pain to be felt. Reduction of acidity by the neutralising effect of alkali or food, or by emptying the stomach through vomiting, then gives relief of pain.

More severe pain will arise where inflammation extends beyond the confines of the ulcer, causing a perigastritis or periduodenitis, and is then continuous and accompanied by persistent abdominal tenderness, with only slight relief from food, alkali or emptying of the stomach.

Appetite in simple ulcer is normally retained or increased unless associated gastritis be present, but food intake may be restricted from fear of pain and some loss of weight may thus ensue. Nausea is not a usual feature. Vomiting may occur as a reflex phenomenon at the height of ulcer pain, giving relief; sometimes from rapid distension of the stomach with blood and resultant haematemesis. Alternatively it may be secondary to obstruction of the food channel through the stomach. In many cases this is temporary from muscular spasm, with or without local inflammatory oedema, a form responding generally to treatment; in some it signifies organic stricture from cicatrisation of the ulcer base. Sensations of acid regurgitation into the mouth and water-brash, with excessive salivation, are very common accompaniments of ulcer.

Constipation is frequent, but a lienteric type of diarrhoea in some subjects is apt to follow the performance of gastro-enterostomy.

Complications of perforation and haemorrhage are dealt with in a subsequent section, but it may be noted here that bleeding of clinical significance occurs in at least half the cases of chronic ulcer. Recurrent fulminating haemorrhage may dominate the clinical picture, or the bleeding may be of less dramatic order, with milder degree of anaemia, from a slowly oozing ulcer.

\section{Physical Signs}

Routine clinical examination may frequently fail to elicit physical signs in cases of peptic ulcer, especially during periods of relative quiescence. Nutrition is usually preserved and anaemia is not a feature apart from blood-loss. Local tenderness is common in the central epigastrium or to either side of the upper abdomen, corre-? sponding with the site of pain, sometimes with cutaneous hyperalgesia, and may be accompanied by muscular rigidity of the upper rectus to a variable degree. Rarely a tender mass may be felt where acute inflammation has followed a slow leak through the ulcer base, and may be confused with carcinoma. Obstruction will outline the distended stomach or portion thereof, with peristaltic waves of characteristic type. Splashing is readily elicited in dilatation of the stomach with retention of its contents, but is of no pathological significance within two and a half hours of a meal.

\section{Special Investigations}

\section{Radiology}

Apart from these sparse findings diagnosis of peptic ulcer depends upon a combination of a careful history and of special investigations. Of these radiology takes the lead. In good hands, a high proportion of ulcers should be demonstrated by means of an opaque meal. Thus it should be possible to diagnose some 90 per cent of gastric, and 75 per cent of duodenal ulcers; the difficulties and errors being higher, the deeper and less accessible the site. Of anastomotic ulcers perhaps the 
majority will be revealed. Despite the most careful radiological examination fallacies will sometimes occur, and the rule for the clinician should be to accept a diagnosis of peptic ulcer, particularly duodenal, based on strong clinical evidence in the absence sometimes of X-ray confirmation. The same comment applies with regard to tests of cure.

\section{Gastric Analysis}

Support for radiological evidence is given by chemical laboratory tests, in chief by gastric analysis. Examination of a single specimen of gastric contents after a one-hour interval is a method of little value and of historic interest only, the fractional test meal being universally employed. As with X-ray examination, it is best carried out in the early morning and the patient's last meal must have been not later than 8 p.m. the previous evening, all drugs being omitted on the day of the test. The fasting stomach contents or "resting juice" are completely withdrawn after the swallowing of a Ryle's tube, the meal prepared from fine oatmeal gruel is then given, and samples of about ro to 15 c.c. of gastric contents withdrawn at regular intervals, preferably half-hourly, for a total of $2 \frac{1}{2}$ hours, by which time the stomach is normally empty.

The specimen of greatest importance is the resting juice; this is examined for volume, odour, consistency, presence of blood, mucus, and food residues, and microscopically for red cells, pus cells, epithelial and malignant cells, with chemical estimation of free and total acidity. Similarly each specimen is examined for volume, total acidity, free $\mathrm{HCl}$, mucus, bile, blood and starch, and the results recorded graphically upon a chart.

The interpretation and significance of the test meal may be briefly described: In uncomplicated gastric ulcer the curve may either be normal or show a hyperchlorhydria. Sometimes an initial low acidity is found from associated gastritis, the production of acid being increased after gastric lavage. In duodenal ulcer it is the rule to find a marked hyperchlorhydria from irritability and hypersecretion of the stomach, irrespective of the phase of activity of the lesion. The resting juice also is usually highly acid.

Two types of curve can commonly be distinguished. In the first, or "climbing" curve, after an initial fall in acidity due to dilution of the resting juice and fixation of free acid by the meal, there is a gradual rise in its level throughout the whole period of observation. Slow emptying of the stomach from pylorospasm, with delayed bile reflux and little or no regurgitation from the duodenum causes a continued rise in the level of
$\mathrm{HCl}$ past the $2 \frac{1}{2}$ or 3 hours period. In the second or "hurry" type of curve a high initial acidity of resting juice after a slight temporary drop rises sharply to a still higher plateau level, which is either sustained or shows a premature fall within an hour or so, due to rapid emptying of the stomach unimpeded by pylorospasm.

In the interpretation of these findings it must be remembered that while many cases of carcinoma of the stomach show an absence of hydrochloric acid, its presence in normal amount or even in excess by no means excludes a diagnosis of malignancy. Other lesions apart from gastric may of course be accompanied by achlorhydria. Conversely a complete lack of $\mathrm{HCl}$ production in a test meal which includes the giving of histamine is strong evidence against benign ulceration.

As mentioned in considerations of treatment, the healing of the ulcer has little effect upon the acid secretory levels of the gastric juice; this indeed is one of the potent factors in recurrence. Reference is made later to the means whereby effective neutralisation of the gastric acidity may be measured.

\section{Occult Blood Test}

Bleeding detectable only by chemical test is $\vec{\emptyset}$ sufficient evidence of active ulceration, grante遰 certain well-known safeguards in technique ang. interpretation, such as the exclusion of haemo? globin- and chlorophyll-containing foods and of bleeding from other alimentary sites. A positive occult blood test or benzidene reaction in the stools then indicates a breach of surface epithelium. The test is so sensitive that slight or doubtful reactions may be ignored. The disappearance of occult blood from the stools is useful evidence of healing of an innocent ulcer and makes malignancy improbable.

\section{Gastric Aspiration}

Gastric aspiration, apart from a test meal, is a measure of value both in diagnosis and treatment. In the normal empty stomach there should be not more than 25 c.c. of resting juice; an excess indicates gastric irritability and hypersecretion, and becomes marked in the event of obstruction of outflow, o whether of spasmodic or organic nature. In these circumstances many ounces, rarely pints, of residual stomach contents may be found, perhaps of dirty malodorous fluid containing mucus, blood, stale food or malignant cells, and of high acidity from foreign acids due to putrefactive organisms. Routine aspiration of such fluid and subsequent lavage with $\mathrm{O} . \mathrm{I}$ per cent $\mathrm{HCl}$ will do much to allay the accompanying gastritis and relieve the 
obstruction present. Even in non-obstructive lesions the removal of highly acid gastric juice by aspiration is a measure of value, as noted later.

\section{Gastroscopy}

Direct inspection of the stomach by gastroscopy is fast becoming the most valuable single measure in diagnosis and in assessment of progress of peptic ulcer. It has been rendered possible by the introduction and development of the flexible gastroscope by Schindler in Germany twelve years ago, and particularly by the improvements of Hermon Taylor in this country. With premedication and local anaesthesia, combined with skill and experience of the operator, the examination is not unduly uncomfortable to the patient. Its value is, of course, greater in the case of gastric than of duodenal lesions. While not infallible, the increased accuracy of diagnosis which it affords should make it a routine measure in the investigation and control of gastric disease. It in no way replaces or diminishes the importance of radiology, but provides an additional and more exact means of determining pathological changes in the stomach and their course under treatment.

Benign gastric ulcers usually show sharp, crater-like margins and a smooth or but slightly irregular yellowish-white floor, though after haemorrhage it may be brownish or dark-red in colour. The adjacent mucosa is often normal in appearance, but may show local inflammatory swelling around the margins of the ulcer. Coincident gastritis produces an oedematous dull mucosa with sticky adherent mucus or mucopus, and sometimes petechial submucous haemorrhages, indicating the need for gastric lavage. Rarely the likelihood of impending haemorrhage may be suspected from an exposed or oozing vessel in the ulcer base. As the ulcer heals it becomes shallower, with cleaning and granulation of the base and subsidence of local oedema at its margins, and a converging stellate pattern develops in the surrounding mucosa from contracture around the ulcer floor.

Too comprehensive a survey must not be expected from gastroscopy, for some areas of the stomach, chiefly the fundus, and sometimes the pre-pyloric region, are inaccessible to direct vision. Its greatest value lies in the determination of progress of a given gastric ulcer, through all stages to complete healing, and in limited measure in the diagnosis from malignant disease.

In the differentiation of benign from malignant ulcers gastroscopy is of much value in skilled hands, but features of malignancy are not necessarily conclusive at a single observation. A carcinomatous ulcer usually has more rounded edges, less well demarcated from the mucosa; its floor is commonly irregular with nodular prominences or ridges, and is more frequently of a reddish brown or dirty grey colour than yellow. In contrast with the excavation of the simple ulcer, it is usually elevated above the surrounding mucous membrane, which may, like the ulcer itself, show nodular irregularities.

Size alone is no guide; a small ulcer may be malignant and a benign reach a diameter of several inches.

It follows, therefore, that a single examination is often inconclusive, and no final decision as to malignancy or otherwise should be sought therefrom. The value of gastroscopy lies rather in serial observation. Wherever the malignancy of a gastric ulcer is in question, gastroscopy should be repeated after a period of three weeks' intensive medical treatment by complete rest, dieting and alkalis. An innocent ulcer will certainly during this time decrease in size and show other evidence of healing. If, on the other hand, the appearances of the lesion are unchanged, it is right to regard it as probably malignant and to treat the patient, if otherwise suitable, by subtotal gastrectomy. In some cases the later gastroscopy will reveal that the ulcer has extended and become more nodular and infiltrative, and in these its malignancy will not be in doubt. Wherever therefore symp-o toms of peptic ulcer are unrelieved or inadequately relieved by medical treatment, gastroscopy shouldo be carried out.

Malignancy apart, in simple ulcer the surest evidence of healing is given by periodic gastroscopy. Caution is taught by the observation that weeks or months after the patient is symptomfree and X-ray findings are negative, the ulcer may still be present as a shallow crater with smooth floor and uninflamed margins. Recurrent bouts of pain, thought clinically to be due to fresh ulceration, are revealed as no more than the lighting up of an ulcer which has never completely healed. It follows then that where an ulcer is within the range of vision it should be gastroscopically controlled until it is known to be healed, and that any fixed duration of hospital treatment and dieting is a bad routine and not necessarily adequate. Where, moreover, it is shown by gastroscopy that an ulcer persistently fails to heal by these means, the indication is clear for surgical treatment.

The duodenum, unfortunately, remains cloaked from view, and diagnosis of ulceration here must rest upon means previously described. Gastroscopy, nevertheless, is not without its value, especially in decisions of appropriate treatment. Degree of hyperacidity bears a close relation to the total acid-secreting surface of the stomach and hence 
to the degree of rugosity of the mucosa. Where the folds are markedly deep and numerous, the hyperplastic type, difficulties and delay in healing by conservative treatment may be expected, and considerations of surgery apply. Simple shortcircuit procedures in such a case are likely to be followed by jejunal ulceration, and good grounds are present for a choice of subtotal gastrectomy.

Jejunal ulcer may sometimes be diagnosed by gastroscopy, which is, however, technically difficult and often affords but a partial view of the stoma.

\section{Clinical differentiation of Innocent and Malignant Gastric Ulcer}

Some of the points aiding this distinction will have already been noted. Clinical aspects are no sure indication. An ulcer of the stomach beginning after the age of forty should be suspect of malignancy until the converse is proved. Age itself is of untrustworthy significance, however, for carcinoma may occur in the third decade and simple ulcer is not unknown in the sixth or seventh. A prolonged dyspeptic history favours a benign lesion, but carcinoma of the stomach may arise in a patient the subject of long-standing indigestion whether from simple ulcer or any other cause.

Conversely a worsening of symptoms in a known case of innocent ulcer does not necessarily mean a malignant change. Marked loss of appetite, weight and strength are more likely to occur in malignant disease, as is anaemia not due to bloodloss. A palpable swelling, though a late sign, nearly always indicates carcinoma. Early obstructive symptoms also point to malignancy. Haematemesis and melaena may be initial or early symptoms of either disease, but gross bleeding is commoner from a simple ulcer. The continued presence of occult blood in the stools after two or three weeks' full medical treatment which includes recumbency is suspicious of gastric carcinoma, since most cases of simple ulcer lose this sign within that time upon an efficient régime. Conversely, a persistently negative occult blood test suggests that the ulcer is innocent, although no absolute rule applies. The fallacy of relying upon fractional test meal findings has been noted, 50 per cent or more of early gastric carcinomata being accompanied by free $\mathrm{HCl}$ in the stomach, sometimes in excess. On the contrary, a complete achlorhydria after the giving of histamine practically excludes a simple peptic ulcer.

The value of X-ray and gastroscopic evidence has been discussed. It may be noted that ulcers of the greater curvature are nearly always neoplastic, and the farther away the ulcer is from the lesser curvature the greater is the probability that it is malignant. Carcinomatous ulcers tend in X-ray appearance to be more ragged and irregular in outline, the gastric rugae being distorted and interrupted in precipitate manner instead of converging in radial fashion upon the ulcer site as in an innocent lesion. The demonstration of a meniscus sign is practically diagnostic of carcinoma. The final judgment of serial $\mathrm{X}$-ray and gastroscopic investigation has been emphasised. If treatment on a medical régime brings about a striking relief of pain and gain in weight, with clearance of the stools, a benign ulcer is likely, but even this is not absolute evidence, since the improvement of a gastritis in cases of carcinoma by rest and dieting will often lead to a temporary regain of appetite. The value of the visual check, therefore, needs no further emphasis.

\section{Chapter II}

\section{Treatment of Chronic Peptic Ulcer}

\section{General Considerations}

The problem of treatment falls into two stages; firstly the healing of the ulcer, and secondly the prevention of recurrence, and of these the first is the simpler task. The treatment of an uncomplicated chronic peptic ulcer is fundamentallp the same wherever its position may be. Therapt is essentially medical, and surgery where indicate is undertaken for specific complications, which are considered later. The over-riding principle in the medical treatment of ulcer is to treat the patient and not solely the lesion.

The importance of the general well-being and of complete bodily and mental rest may easily be overlooked by considerations of detail in diet and drugs.

Any ambulant treatment at the outset of the illness is unsatisfactory and wasteful of time, few chronic ulcers healing while the patient is up and about. A minimum of four to six weeks' complete bed-rest is essential, followed by a like period of quiet convalescence where progress is satisfactory. It $₹$ is often difficult to convince the patient of the $\frac{\text { ? }}{5}$ necessity for this strict régime, the more so when $\rightarrow$ he is of the irritable, restless type. Rapid disappearance of pain and early return of well-being $\tilde{N}^{-}$ anxiety over business or economic affairs often $N$ leads to pressure upon the medical attendant to $N_{\omega}$ relax restrictions prematurely. A clear explanation is required that relief of symptoms does not imply that the ulcer has healed.

For these reasons care must be taken to ensure $\stackrel{\mathbb{D}}{\rightarrow}$ for the patient adequate mental relaxation, with 0 quietness, sleep and freedom from worry, mental 
I L L USTRATION S

GASTRICAND DUODENAL

ULCER

R. SLEIGH JOHNSON, M.D., M.R.C.P.

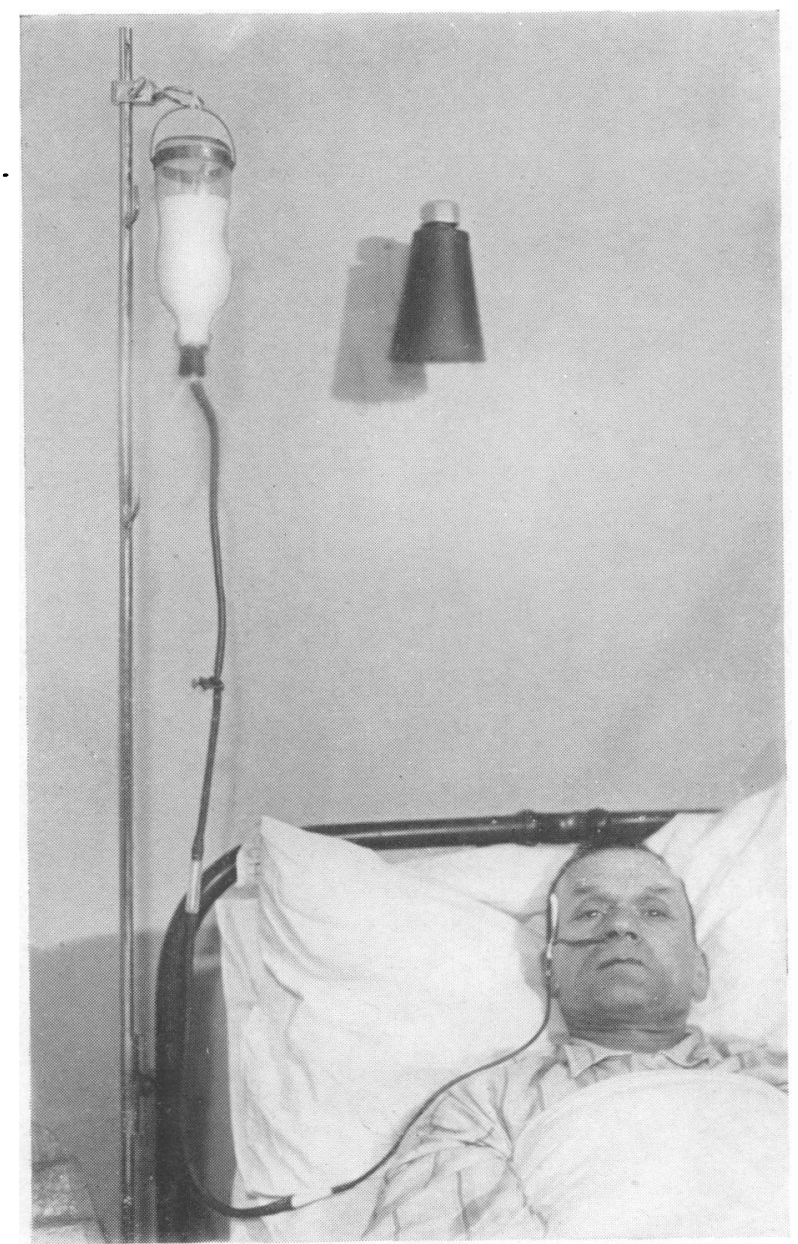

Method of feeding by continuous milk drip. 
rest being no less important than physical. Social adjustments in this will play their part, but sedatives are often required in addition, phenobarbitone being usually of more value than bromides. Needs will vary with the patient's individual type and circumstances, the aim being by encouragement and advice to promote a wholesome psychological outlook. It is common experience that the required régime is more easily and efficiently carried out in hospital than at home.

At this stage of treatment smoking should be wholly forbidden and alcohol avoided. Attention must be paid to the teeth to obtain a clean mouth and adequate facilities for mastication.

\section{Principles of Dieting}

Perhaps too much emphasis in the past has been placed upon the relative merits or demerits of particular schemes of dieting in peptic ulcer, and upon the neutralising of gastric acidity, to the neglect of a balanced diet providing sufficient calories, salts and vitamin requirements. Treatment is necessarily prolonged and nutrition and health must be maintained. Frequency of feeds is at least as important as their nature, granted the exclusion. of obviously unsuitable foods. While it may be true that the presence of hydrochloric acid in excess is the chief factor in maintaining active ulceration, treatment is not solely a simple chemical equation of its neutralisation by appropriate alkalis. Nevertheless it is by combating the effects of acidity that the main therapeutic result is obtained. Drugs, including alkalis, play an important part in control of hyperacidity and are for convenience considered later. Food is the primary and essential buffer in virtue of the acid-combining. power of its foodproteins.

To render this maximal the following principles should be observed:

(a) the food must be soft, smooth, fluid when ingested, and free from coarse elements to avoid irritation of the ulcer area.

(b) the bulk of the individual feeds must be small, and their character bland, to prevent distension of the stomach and excessive secretion of gastric juice.

(c) feeds must be given frequently so that the process of neutralisation is continuous, the total intake of food sufficient to maintain nutrition, and the stomach is not left empty for prolonged periods exposed to acid gastric secretion.

With regard to the second of these principles, it may be noted that the quantitative differences in gastric secretion produced by various foods is less than formerly thought, and that although fats do inhibit gastric secretion and emptying, they play less part in maintaining gastric neutrality than does the acid-combining property of proteinfoods.

The main ingredient of all such diets is milk, itself an efficient alkali, neutralising an equal volume of gastric juice containing 0.3 per cent $\mathrm{HCl}$. The systems of diet first introduced in the Lenhartz and Sippy régimes, based chiefly upon milk and eggs and milk and cream respectively, were each deficient in caloric value and are now seldom used in their original form, though modifications are still in favour in many hospitals, details of which are readily available. A more balanced diet with greater variety is that devised by Hurst, the most widely used scheme, the first stage of which is based upon feeds at hourly intervals alternately of citrated milk, five ounces, and a fruit, vegetable or carbohydrate purée. More recently a still more generous régime has found favour with many physicians in the form of Meulengracht's diet, embracing whole milk, porridge, barley, rusks, cream crackers, fruit and vegetable purées, eggs, fish and even meat; recommended at first for the more generous feeding of patients after haematemesis, it is also used in the ordinary stages of ulcer treatment, and includes quite large and varied meals. The reaction against semi-starvation after gastroduodenal haemorrhage is fully justified, but ing most hands greater safety and better results are obtained from adherence to Hurst's fundamentase principles. Hurst's régime includes a number of drugs, whereas in Meulengracht's scheme these are practically excluded.

The first stage of dieting (the strict ulcer régime) is continued not for any stated number of weeks, but until there is freedom from all spontaneous pain or discomfort, tenderness and muscular rigidity are no longer present, and the stools show a negative test for occult blood on three consecutive occasions. Where pain is severe and resistant, gastric spasm a marked feature, or emptying of the stomach is delayed by a partial obstruction from this cause or from oedema, and further where it is desired to afford the stomach the maximum degree of rest, as after haematemesis or melaena, feeding by a continuous milk drip gives even better results than a first stage Hurst diet. A Ryle's tube made non-irritant with 2 per cent cocaine ointment is passed into the stomach, preferably through the nose, alternatively through the mouth, and fixed in position by strapping to the cheek. Through this, from a height of about two feet, the patient is given a continuous drip of citrated milk, day and night, from a suitable container such as a transfusion bottle, fixed to a stand or bed-extension and adjusted to a rate of about 40 drops per minute (Fig. I). The patient receives 
in this way a total of five pints of milk in the 24 hours, and where desired the caloric value and protein content of the diet may be increased by the addition of glucose and of a ro per cent solution of casein hydrolysate, a pint of the latter being added to the five of citrated milk. Alkalis and other drugs needed may be given either in the drip or separately by mouth, the latter being generally preferred. Most patients will tolerate feeding in this way for periods of up to three weeks. some for much longer. Rest of the stomach is as complete as may be obtained. Appetite secretion is minimised, and the stomach is never left exposed at night or for other interim periods to the action of highly acid gastric secretion, neutralisation of which is made a continuous process. Nutrition is well-maintained, and healing is more rapid by this method in the writer's experience than by any other. An initial soreness of nose or throat for the first day or two is seldom maintained. A period of milk-drip feeding in this way may well be followed by a first stage Hurst diet.

Whichever method be used, when tests have confirmed a satisfactory response, this initial stage of dieting is followed by an intermediate one, in which some of the milk feeds are replaced by lightly cooked and easily digested meals allowing greater liberty of choice. This second stage must be maintained as long as any evidence of active ulceration is present, and may therefore occupy many weeks or months. It is not possible from clinical observations alone to tell when healing has taken place or to assess its degree, for it is not complete until the defect is closed and covered by a normal glandular mucosa; this stage is delayed long after the disappearance of pain and even after vanishing of the crater seen on $\mathrm{X}$-ray pictures.

Most failures of medical measures arise from an insufficient length of treatment. Control by periodic $\mathrm{X}$-ray and gastroscopic examination is required at intervals of three to four weeks, until complete healing can be demonstrated, or the need for alternative treatment decided upon. The value in diagnosis by these means of malignant ulcer has already been mentioned. In duodenal ulcer a greater measure of reliance has to be placed upon radiology alone.

Maintenance of nutrition should be checked by a weekly observation of weight, and where anaemia is present, as after haemorrhage, periodic blood counts are required.

\section{Drugs in the Treatment of Peptic Ulcer}

(a) Acid-neutralisation. Sippy's aim in neutralising hydrochloric acid was to inactivate pepsin, but it is now known that high acidity is in itself more destructive upon an ulcer than the solvent action of the peptic ferment. The object nevertheless remains unchanged to maintain a neutralisation of all free $\mathrm{HCl}$ while food and its accompanying secretion is present in the stomach; it is also necessary, and more difficult, to neutralise the continued secretion of acid gastric juice during the night hours when the stomach is empty of food.

Alkalis are the most widely used as well as the most abused remedies in the treatment of peptic ulcer. In their rapid relief of pain lies their danger in ignorant hands; advertisements of proprietary "stomach-powders" fill the daily press and lull their victims into a false security. Properly used, alkalis are of the greatest value and second only to correct dieting in treatment.

A wide range of ant-acids is available, with their special advantages or otherwise according to the case. Sodium bicarbonate has a quick though short neutralising action, but produces distension of the stomach by liberated $\mathrm{CO}_{2}$ and a marked subsequent secretion of acid, rendering it unsuitable as an alkali for this purpose. In view of its greater solubility it is moreover more prone than are other alkalis to produce alkalosis. The more gradually acting magnesium and calcium salts are fully effective, and may be combined in proportion to regulate the bowels, the former being mildly aperient and the latter astringent. Mage nesium oxide, carbonate and tribasic phosphat are excellent; the trisilicate has come into favour with some, but is a more costly preparation and no more efficient than its fellows. Effective dosage requirement varies with the degree of acidity present; commonly (as in Hurst's scheme) doses of a drachm of one of the above salts in powder form are sufficient, given with water before each of the purée feeds, and one drachm or more of emulsio magnesiae B.P.C. (containing grs. $v$ of magnesium oxide) before each of the alternating milk feeds; a double dose of alkaline powder is given at night. Calcium preparations, in the carbonate or tribasic phosphate, are useful alternatives, and a good case has lately been made for colloidal aluminium hydroxide, in the form of aludrox in similar dosage. Bismuth salts are feeble alkalis in their degree of neutralising power and the idea of their forming a protective coating to the ulcer floor belongs to past days; they are, like calcium salts, mildly constipating.

In applying the continued drip theory the rôles of food and alkali may sometimes with advantage be reversed, and a constant administration of alkali in solution or suspension, e.g. as aludrox, may be given through a Ryle's tube, with hourly or two-hourly feeds by mouth.

Sodium citrate, besides inhibiting rennin and 
preventing clotting of milk, is also an effective alkali and is added to all milk feeds in proportion to two grains to the ounce.

Where hypersecretion is marked or a measure of obstruction present, alkali administration by itself is insufficient to ensure continued neutrality of stomach contents throughout the twenty-four hours, the more so at night. Routine aspiration of the stomach at bed-time, or oftener, should in these cases be carried out, thereby shortening the period during which the ulcer is exposed to the action of the acid gastric juice. It affords, moreover, a means of assessment of correct alkali dosage; if this is adequate, the stomach contents thirty minutes after a dose of the powder should contain no free acid; its presence then is a call for more frequent or larger dosage. In non-obstructive cases it is the rapidly emptying stomach which most needs aspiration at night, food contents and alkali having been quickly passed on, and a collection of highly acid secretion being then poured out into the empty stomach to remain there during the night. The case is clear also for gastric aspiration in obstructive ulcer, whether from pylorospasm or duodenal stenosis, and this should be continued at least twice a day until the residual contents are not more than 50 to 100 c.c.

\section{Alkalosis}

The indiscriminate giving of alkalis in excess, especially when accompanied by vomiting or independent renal disease, may cause a serious disturbance of the acid-base equilibrium and chloride content of the blood, a condition known as alkalosis. This is most often seen where a continued depletion of chlorides has occurred through low salt intake and vomiting, especially with pyloric obstruction, benign or malignant. Symptoms include irritability, lassitude, mental confusion and headache, with loss of appetite, nausea and distaste for milk, followed by profuse vomiting and drowsiness, and in serious cases the development of a state closely simulating uraemia, with low output, albuminuria and high blood-urea, perhaps progressing to coma and tetany. The chloride content of the blood is always greatly lowered. Treatment called for is the immediate stopping of alkalis and the giving of large amounts of sodium chloride as normal saline solution intravenously, with glucose and abundant fluids by mouth, supplemented where it can be taken by oral administration of sodium chloride and ammonium chloride in capsules in large doses. If tetany occurs intra-venous calcium gluconate is given. Prophylactically, in conditions where alkalosis is a likely complication three to five grammes of sodium chloride may with advantage be added to the daily milk ration. (b) Inhibitory Drugs. Acid neutralisation by alkalis is commonly supplemented by measures. claimed to diminish the secretion of gastric juice, and of these belladonna or its alkaloid atropine is the most used. Its action in restraining gastric secretion is doubtful, but its effect as an antispasmodic is more certain, and it is hence of definite value in inducing relaxation of spasm at the pylorus or at the site of ulceration. It is given as routine in the Hurst's diet in doses of I/IO0-I/I50 gr. in a drachm of water by mouth before two of the daily feeds, and a double dose before the Io p.m. feed, and more may be given if spasm persists, short of producing blurring of vision and dryness of the mouth. As evening and nocturnal secretion is the most difficult to check, late afternoon or bed-time is the time of choice.

Fats in general inhibit gastric secretion. Olive oil or in war-time arachis oil taken twice daily before meals in ounce doses has this effect besides forming a valuable food. The addition of cream to the milk feeds is of similar benefit.

(c) Sedatives.-The indications for and value of sedative drugs in moderate doses in the quietening of the mental state have already been mentioned.

(d) Haematinic drugs. Anaemia of some grade from slight blood-loss over a long period is so common in peptic ulcer that regular blood investigation should always be made. Where haemoglobin deficiency is found, iron is indicated inc liquid form as iron and ammonium citrate or colliron in appropriate dosage; after frank haemorrhage early and massive iron administration is required, begun as soon as the bleeding has ceased. In view of the low iron content of ulcer diets, a minimum of five grains of a ferrous salt per day as supplement is a good routine, despite the absence of demonstrable anaemia, for all patients upon the restrictive stage of dieting.

(e) Vitamins. The most likely deficiency in this regard arises from the limitation of fresh fruits and vegetables, and a long-continued ulcer diet should always be supplemented by Vitamin $B_{1}$ 2,000 units and Vitamin $C$ as ascorbic acid Ioo mgm. given daily. There is no objection to the giving as well of fresh orange or other fruit juice.

(f) Control of bowels. Straining at stool is obviously undesirable, particularly if recent bleeding has occurred. Where regulation of the diet and additional magnesia do not suffice, agar and liquid paraffin preparations will help towards an easily passed stool.

(g) Histidine. The injection of histidine products recently had a short-lived vogue in the medical treatment of peptic ulcer. It has since been shown to be entirely unsupported by evidence or by results, and its use has now been discarded. 


\section{After-care of Peptic Ulcer}

\section{"Post-ulcer" régime}

The plan of treatment outlined is maintained either so long as any evidence of active ulceration remains, or until the conclusion is reached that medical means alone are inadequate after a prolonged trial. Where healing is established, the patient is allowed up by stages and progresses on to a post-ulcer régime of diet and habits, which he is instructed to follow for a minimum of two years or if possible permanently. From the instructions he will be taught to maintain small frequent meals with intervals of not more than two hours, to adhere to soft readily digested foods and avoid hard, irritant or highly seasoned articles with indigestible residue. Above all he will be advised to obtain ample time and regularity for his meals, to rest before and afterwards, and to avoid excessive fatigue and as far as possible intercurrent infections. He must pay proper regard to his teeth and his bowels and continue to take an alkaline powder after each of his main meals. Smoking and alcohol are best avoided altogether. Any return of symptoms calls for a prompt resumption of bed-rest and strict dieting.

Could this ideal or even pious advice always be followed, the prognosis of medical treatment of peptic ulcer would be much better than it is. Prospects of remaining well vary greatly with social status and economic means. The patient in comfortable circumstances may be able to continue the whole régime religiously and remain free from symptoms, with a careful diet and sheltered life quite impossible for a working man with living to earn and family to support. The occupation followed may be a stumbling-block and its type is not easily changed. Even granted the best of after-care, no guarantee can in fact be given of freedom from recurrence of ulceration. Strong argument obtains therefore, for the indefinite following-up of all ulcer patients in clinics organised for the purpose, supported by X-ray and other special investigations as required.

\section{Indications for Surgical Treatment}

The best efforts of medicine in this disappointing disease are not always blessed with success. It is pertinent therefore to consider what are the indications for surgical treatment. Some will be obvious and are by nature emergency procedures; others should be embarked upon only after carefully weighing the pros and cons of individual circumstances. (a) Failure of Medical Treatment

This is the most frequent reason for operation; it presupposes that medical treatment should have been really adequate, and not a half-hearted trial. It more commonly applies in intractable duodenal ulcer than in gastric, and apart from any complications of the dramatic order.

The type of patient with prolonged history of pain interspersed with increasingly frequent and severe relapses is not best served by repeated vain attempts at medical cure, but should if otherwise fit be treated surgically to avoid a lifelong dyspepsia. Within this group will be many with deep penetrating ulcers embedded in callous scar tissue and adherent to other organs. In some cases the failure to heal of a gastric or jejunal ulcer after gastro-enterostomy will be an indication for more radical surgical treatment.

The trend of opinion in these medical failures is increasingly in favour of partial gastrectomy, but in choice of surgery the degree of hyperplasia and hypermotility of the stomach will be a factor. Suitability for operation on grounds of age, pulmonary or cardio-vascular condition has of course to be considered.

(b) Inability or unwillingness to maintain a full measure of efficient medical treatment.

Lack of co-operation on the part of the patient will often indicate the advisability of surgeryô. This is sometimes concerned with psychologicaF type and temperament. Frequently, however, it is due to economic reasons, nature of occupation, pressure of time or domestic circumstances out of his control; it may be essential in such a case to restore if possible the ability to pursue a heavy or exacting type of employment, the prospects of which depend upon successful operation alone.

\section{(c) Recurrent haemorrhage.}

Most of the fatalities from bleeding follow repeated blood-loss. While the immediate treatment of ulcer-haemorrhage is by general agreement medical, it is certain that recurrent haematemesis should be followed by operation as soon as the patient's general state permits.

\section{(d) Suspected malignancy.}

The failure of a gastric ulcer to respond to full medical treatment within at most a few weeks indicates the advisability of exploration, to confirm diagnosis and where suitable to carry out a subtotal gastrectomy.

\section{(e) Perforation of the ulcer.}

This is an obvious need for immediate surgery. 


\section{(f) Organic obstruction.}

Obstruction which is largely due to oedema and spasm will frequently be relieved by dieting, antispasmodic drugs, aspiration and lavage. Where, however, the obstruction is of organic origin, as in hourglass stomach, duodenal or pyloric stenosis, surgical treatment alone can relieve it.

\section{REFERENCES}

HURST, A. (I936), Post-Graduate Surgery (Ed. R. Maingot), 1, 573. MORLOCK, R. (I 944), Proc. Staff Meeting Mayo Clinic, 19, 449

SIPPY B. (I9I5) $A . M . A$ 64, 1625 .

WINKELSTEIN, ASHER, CORNELL, ALBERT and HOLLANDER (1942), J.A.M.A., 120, 743.

\section{X-RAY APPEARANCES OF GASTRIC, DUODENAL AND JEJUNAL ULCER}

\author{
By H. C. H. Bull, M.A., M.B., B.Ch., \\ M.R.C.P.(Lond.) \\ (Hon. Radiologist Southend General Hospital)
}

\section{Gastric Ulcer}

A gastric ulcer is first an erosion of the mucous membrane which subsequently penetrates the deeper layers of the submucosa muscle and peritoneum. Radiology is shadow diagnosis and so the burrowing and eroding ulcer becomes to us a niche or projection from the clearly defined stomach wall demonstrated in shadow by the contrast medium filling it.

Of gastric ulcers $90 \%$ are found on or about the lesser curvature above the incisura angularis. The lesser curvature differs anatomically from the rest of the stomach and is the direct pathway of food and fluids entering, but just why it is the seat of ulceration is not known.

Of the remaining 10\% some are found on the lesser curvature between the incisura and pylorus; the others-and they are few, probably less than $\mathrm{r} \%$-are on the anterior wall of the pyloric end of the stomach.

Simple ulcers have been recorded on the greater curvature. I have not seen one other than a malignant ulcer, but there are a few published cases.

The outstanding fact and diagnostic feature-the "direct evidence" of gastric ulcer-is the niche seen in profile or tangential view projecting outwards from the cavity of the stomach illustrating in shadow picture the identical form seen in postmortem pathology (Fig. I).

These gastric ulcers may be small or large, recent or of long standing. The size is some estimate of chronicity, but any size above one inch diameter carries with it a suggestion of malignancy. Despite the work, pathological and surgical, devoted to study of the early malignant changes in gastric ulcer there is direct evidence that chronic gastric ulcers often develop malignant change. When large the ulcer is usually old and certainly penetrating in the sense that it has broken through and passed beyond the natural boundaries of the stomach. Once an ulcer has reached the peritoneum, established adhesions between it and an adjacent viscus, usually the pancreas or the liver, and broken down the peritoneal boundary, erosion proceeds rapidly and the crater becomes a large cavity of irregular outline. Such an ulcer tends to show a relatively narrow passage at the point of exit through the peritoneum, expanding into a large cavity beyond.

In shape gastric ulcers vary a good deal according to chronicity and position. The small ulcer projects from the lesser curvature as a blunt point or a rounded bud and its outline is relatively smooth. The larger ulcer which has penetrated through the peritoneum will often show a fluid level with a gaso bubble above when the patient is in the erect? position.

An ulcer situated between the incisura and pylorus on the lesser curvature has the same general characters as that above the incisura but, as gravity plays a part, it does not hold barium so well in the erect position. Examined under the screen, barium can be pushed up into the crater, but when the pressure is released a certain amount only sticks on to the rough surface, and is better demonstrated on the films taken with the patient prone.

Carman, in Igizo, described the saddle ulcer lying astride the lesser curvature between incisura and pylorus, an ulcer with rolled edges holding gutters of barium when pressure was applied so that the appearance was that of detachment. To this he gave the name "meniscus" and he said it was always malignant. Such an ulcer is not common. Even a simple ulcer often has thick rolled edges, thus retaining barium, and it has been my experience that an innocent ulcer can show the meniscus sign. If we add to Carman's dictum that the meniscus sign in an ulcer of one inch or more diameter spells malignancy, then we are approaching nearer the truth.

Thick rolled edges, to which mention has been made in connection with malignancy, is a feature 